\title{
A summary of LED lighting impacts on health
}

\author{
Cosmin Ticleanu, ${ }^{1, *}$, Paul Littlefair ${ }^{1}$ \\ ${ }^{1}$ Building Research Establishment (BRE), Bucknalls Lane, Watford WD25 9XX, UK
}

Received 5 June 2015, Revised 31 July 2015, Accepted 12 August 2015

\begin{abstract}
Lighting can affect the health of people in buildings. This goes beyond the safety aspects of providing enough illumination to see by; lighting affects mood and human circadian rhythms, while poor lighting can cause glare, headaches, eyestrain, aches and pains associated with poor body posture or, in extreme cases, skin conditions and various types of sight loss. These aspects ought to be considered by designers and building owners and occupiers in order to improve the lit environment and use adequate lighting and lighting controls that meet the recommendations of codes and standards. Various types of lighting can have different impacts depending on their spectral, optical and electrical characteristics. This paper discusses potential impacts of LED lighting on human health, and is based on a recent BRE review of research investigating the most typical effects of lighting on human health.
\end{abstract}

Keywords: light and health, LED glare, LED flicker, melatonin supression, LED skin exposure.

\section{Introduction}

Lighting directly influences the performance of visual tasks by making critical details of tasks more or less visible. Poor lighting can make it hard to see clearly and may contribute to slips, trips or falls, whilst too much bright light can cause dazzle and mask otherwise obvious hazards. Insufficient light, high illuminance or luminance contrasts between and across working surfaces, glare, veiling reflections, shadows and flicker, can all make visual tasks difficult to perceive appropriately. These difficulties lead in turn to visual discomfort which may manifest itself through the occurrence of red, itchy eyes, headaches, and aches and pains associated with poor body posture [1]. Additionally, lighting can affect the health of people in buildings [2]. This goes beyond the safety aspects of providing enough illumination to see by. Lighting affects mood and human circadian rhythms; and poor lighting, besides causing visual discomfort and associated effects, can, in extreme cases, lead to skin conditions or various types of sight loss. Lighting using light-emitting diodes (LEDs) is relatively new compared to other types of lighting, and it is important to consider any potential impacts on the health of building occupants whenever LED lighting is installed. Being directional by nature, LEDs can be very bright and glaring. Some very bright LEDs may cause retinal damage if viewed directly. LEDs can exhibit flicker, which can cause headaches, eyestrain or epileptic seizures in some people. Night-time exposure to bright sources rich in blue light, such as LEDs, can alter the body clock and lead to various health problems. LEDs also have

\footnotetext{
* Corresponding Author: Cosmin Ticleanu, Tel.: +44 7404404293.

E-mail address: Cosmin.Ticleanu@bre.co.uk
} 
benefits compared with other conventional types of lighting, as they do not contain mercury and have lower human toxicity potential, emit little or no UV and do not generate electric or magnetic fields that can damage human health. These aspects ought to be considered by designers and building owners and occupiers in order to improve the lit environment by using adequate lighting and lighting controls that meet the recommendations of codes and standards.

\section{Glare from LED lighting}

Human eyes can adapt to a wide range of light levels from almost total darkness to very bright scenes. However, comfortable vision is possible at any one time only within a limited range of light levels. Excessive light levels and luminance contrasts can lead to glare. Bright areas within the visual field, particularly when their luminance is much higher than the ambient average, can create glare. Glare can be experienced as disability glare or discomfort glare [1].

Disability glare affects the ability to see and leads to some degree of temporary loss of vision, and is produced by high luminances in a lower luminance scene typically by point light sources such as bright spotlights aiming at the observer's eye, or by large area sources such as a bright luminaire. Disability glare does not itself pose direct health risks, but can lead to indirect effects by weakening vision or the ability to recognise objects, and thus poses obvious safety risks for specific activities. Disability glare can be avoided by the correct aiming of light towards areas of interest, as well as by the use of suitable shielding against direct view of light sources and the high luminance parts of luminaires.

Discomfort glare appears in the form of visual discomfort, annoyance, irritability or distraction without affecting the ability to see, but leading to symptoms of visual fatigue. It is the most common form of glare from lighting [3] and can cause eyestrain and other forms of discomfort such as neck- and backache [4, 5]. It has also been found that discomfort glare can adversely affect mood and wellbeing [6]. Depending on lighting conditions, the visual task and individual sensitivity of observers, symptoms typically include irritation (e.g. inflammation of the eyes and lids); dry or watery eyes; itchiness; tense muscles; breakdown of vision, blurred or double vision; headaches or fatigue. Such symptoms can further lead to discomfort and stress [7]. Discomfort glare in interior lighting is quantified by the Unified Glare Rating (UGR), with maximum UGR limits given by standards for various applications that typically range from 13 to 28 .

LEDs are typically small, directional light sources, which can be very bright and glaring unless they are appropriately positioned or incorporated within non-directional luminaires that contain shielding elements or diffusers appropriately designed to reduce or avoid glare. Diffusing LED panels may be a source of glare if they appear bright when viewed from a shallow angle. Sometimes retrofit tubes may cause glare because of the optics of the luminaire in which they are inserted. Glare from LEDs installed in buildings can be direct or reflected in glossy surfaces such as a computer screen or shiny display walls, and LED luminaires need to be set up to avoid a direct view of the lamp by building occupants. Despite the directional character of LED light sources, which makes them very bright, LED luminaires have become available that have UGR values within the ranges recommended for various applications. LED panels can be equipped with diffusing panels that limit their UGR index at 19, making them suitable for office lighting, although this usually causes a loss in light output.

\section{Optical hazards from LEDs}

Whilst glare does not itself necessarily cause direct damage to the eye, exposure to too bright light, besides leading to glare, may cause particular damage to the eye. The type and extent of damage depends on the brightness (luminance) of the light source, its angular size (which partly depends on how far away it is), its spectrum and the duration of exposure.

Generally, short wavelengths (UV and blue) are the most damaging. The cornea, conjunctiva and lens are most sensitive to UV radiation. The retina is more likely to be affected by blue light, since UV radiation tends to be absorbed by the rest of the eye, particularly the cornea and lens, before it reaches the retina. Only about 1 to $2 \%$ of the longer wavelength UV radiation, UVA, reaches the retina. 
Although LEDs emit little or no UV radiation, they can form a very bright light source, and some types of high power LED can cause retinal damage if viewed directly. Depending on the LED luminance, angular size and spectrum, and the duration of exposure, retinal damage can take various forms, from photoretinitis and retinal burns to macular degeneration [2]. Cree [8] has published risk group data for their X Lamp series of LED sources. Most were in risk groups 1 (low risk) or 2 (moderate risk), with one LED (a high power royal blue lamp) in risk group 3 (high risk). Lamps in this risk group would need to be screened in use so that they could not be viewed directly. A Spanish study [9] reported damage to human retinal cells from three colours of LEDs (red, green and blue) but their experiments were carried out at extremely high illuminances (around 34,000 lux, around 100 times typical indoor levels).

\section{LED flicker}

Flicker is generally defined as a rapid and repeated change over time in light brightness. The eyes are particularly sensitive to flicker, which is mainly perceived towards the edges of the visual field. In general, depending on individual sensitivity, flicker can have effects ranging from visual discomfort, fatigue and decreased visual performance to the onset of some forms of epileptic seizures $[4,10]$.

Critical flicker frequency is the threshold above which flicker can no longer be perceived by human eyes. Research has found that in general flicker can be directly perceived by human eyes at frequencies of $60 \mathrm{~Hz}$ and lower [11]. Below $60 \mathrm{~Hz}$, flicker can cause headaches and eyestrain, or even photosensitive epileptic seizures in those who are susceptible [12]. Above $60 \mathrm{~Hz}$, flicker is generally too rapid to be seen by most people, although some studies have found that long-term exposure to higher frequency flickering can lead to potential health problems, such as headaches and eyestrain [13]. Even at frequencies well above critical flicker frequency, nonperceived flicker may have effects on visual performance or induce symptoms of visual fatigue, eyestrain or headaches [14]. Electroretinogram measurements have found that flicker in the range 100 to $160 \mathrm{~Hz}$ and even up to $200 \mathrm{~Hz}$ may be non-visually detected by the human retina [10]. Flicker can still have negative effects even when people who are sensitive to flicker are not actually aware of their sensitivity or that the lighting responsible for their discomfort is flickering [15].

Whilst most conventional electric light sources flicker due to regular fluctuations in the Alternating Current (AC) supply, flicker characteristics can be found in many commercially available LED sources especially when paired with existing lighting control systems in a retrofit situation. LED flicker characteristics mainly depend on the LED driver, and dimming an LED can induce or increase flicker, particularly when using phase-cut controls or pulse-width modulation [15]. In general, flicker can be minimised by ensuring supply stability or by using high-frequency electronic control gear that produces a reduced percentage modulation in light output. AC LEDs are more susceptible to output variations due to power surges and voltage fluctuations. LED drivers of acceptable quality, however, employ high-frequency switching power supplies that attenuate the AC component of the mains supply at the output and thus help reduce flicker. Flicker can still occur when dimming LED lamps by means of mains voltage dimmers and drivers. Direct Current (DC)-based LED drivers are available that can dim the light output of the LED lamps to less than $1 \%$ without using pulse width modulation, so avoiding the risk of flickering from light output fluctuations [16].

LED lighting can exhibit flicker at higher modulation than conventional fluorescent lighting, which may also have health effects at higher frequencies [10]. It has been observed that point light sources are less likely to induce seizures and headaches than a diffuse light source. Hence flicker from LEDs used for general lighting such as LED panels appears to have more negative effects than small LEDs used in instrument panels. Moreover, impacts from invisible flicker tend to be stronger in case of visual tasks requiring precise positioning of eyes, such as when reading, and large LED display boards viewed from close proximity may induce seizures in people suffering from photosensitive epilepsy [10].

\section{Night-time exposure to LED lighting}

Due to the powerful entrainment effect that light has on human circadian rhythms, exposure to artificial light at night impacts on the circadian clock by pushing it back over time. When circadian rhythms are synchronised with the solar day, they cause alertness during the day and sleepiness during the night. When they are desynchronised due to factors such as artificial light at night, jet lag or shift work patterns, alertness and 
sleepiness may occur at the wrong times, and sleepiness may not correspond with the time designated for sleeping. If the light experienced at night is brighter, it will have more impact on the body clock, which is designed to respond to light from the sun. However, any type of light can do this; it is not something which is specific to LEDs compared to other light sources.

The light level in the evenings before bed time is important to sleep quality [17]. Whereas the body's level of melatonin is frequently used as a biological marker for circadian impact, the minimum level of light which can result in a change is still being studied. In an experiment by [18], exposure to light levels of more than 200 lux in a room before bedtime was found to have a measurable effect on melatonin levels compared to a group exposed to dim light of less than 3 lux. The amount of light generated by a self-luminous tablet computer over two hours is enough to trigger a change in melatonin levels, although this does depend upon the type of task being performed by the tablet, as illuminances can range from 5 to over 50 lux depending on task [39]. Light from a television was not found to have the same effect [20]. [21] suggest that a reasonable and conservative working threshold for suppressing melatonin by light at night would be about 30 minutes exposure to 30 lux at the eye for a white light source. However, any given threshold value would vary with the spectral irradiance of different light sources. The spectral composition of a light source affects the degree to which it may impact on sleep. If a light source generates a lot of its light in the blue wavelengths, commonly taken between 400 and 500 $\mathrm{nm}$ [22], this will have a greater potential to keep us awake since blue light appears to affect alertness, body temperature and heart rate [23]. Suppression of melatonin, a key marker of circadian rhythm, occurs most strongly between 446 and $477 \mathrm{~nm}$ [24] meaning that this particular light source would be likely to elicit alertness, but also to potentially shift circadian rhythms and affect sleep if experienced at night. A common type of cool white LED chip which comprises a blue light with a yellow phosphor coating produces significant light output in the 460 to $500 \mathrm{~nm}$ range.

One of the strengths, and complications, of LEDs is that they can be manufactured to generate light in very specific areas of the spectrum, even in a light which appears white to the naked eye. This means that LEDs will all have their own slightly different spectral distribution, and only basic generalisations are possible. Light, including blue light, at the wrong times has been associated with a number of long-term health effects. Czeisler [25] states that because solid-state white light is typically rich in blue light, and the intrinsically photosensitive retinal ganglion cells of the retina are most sensitive to blue and blue-green light, night-time exposure to LEDs is typically more disruptive to circadian rhythms, melatonin production and sleep than incandescent lighting. However, blue light from LED sources during daytime can also be used to correct disrupted sleep [26] and provide a countermeasure to desynchronised circadian rhythms or diminished alertness [24]. Studies by Glickman [27] and Strong [28] compared blue and red LEDs in light therapy treatment. The blue light was found to be more effective than the red light at reducing the symptoms of SAD. The findings support the idea that shorter wavelength visible light (450 to $500 \mathrm{~nm}$ ) is key in the treatment of SAD.

\section{LED content of toxic chemicals}

Early LEDs were made from gallium arsenide and emitted invisible infrared radiation. The first commercial red LEDs made from gallium arsenide phosphide appeared in the late 1960s, green LEDs made from gallium phosphide appeared in the 1970s, but blue LEDs made from gallium nitride appeared only in the 1990s owing to the technological hurdles that first had to be overcome. Current LEDs are made from a variety of semiconductor materials that combine gallium, aluminium and indium with arsenic, phosphorus and nitrogen. Silicon carbide and sapphire can be used as substrates for blue LEDs to improve their efficiency.

Various research studies [29, 30,31, 32] have investigated the toxicity of different metals contained in electric lamps as potential hazardous waste. However, individual substance content was estimated at the end of life by life cycle analysis and potential health hazards due to direct toxic effects from the metals when operating the lamps, if any, were not considered. By excluding emissions associated with the use phase, life cycle analysis performed by [29] shows that an LED lamp contains substances that have a human toxicity potential 45 times higher than those in a classic incandescent lamp, and 3.3 times higher for a CFL. However, by also including the use phase and normalising the results for light output and lifetime, [30] found that incandescent lamps have significantly higher human toxicity potential compared to LEDs due to toxic emissions that are mostly caused by energy consumption. A similar study [31] also found that an LED lamp and an LED luminaire had around 45\% and $35 \%$ of the human toxicity potential of the incandescent lamp, respectively. On the other hand, most 
materials used in LED lighting products can be recycled or recovered at the end of life. Another study [32] found that arsenic, copper, nickel, lead, iron and silver as detected in various LEDs analysed would be of greatest concern for human health. Overall, the white LED analysed would be the least harmful to human health in terms of content of toxic chemicals, due to the low copper content and absence of arsenic and lead, whilst the low-intensity red LED analysed would not comply with the EU RoHS Directive [33]. The authors [32] also suggest that accidentally inhaling vapors emitted by a broken LED lamp would not be likely to cause lethal diseases, but recommend care and protection when sweeping up the debris.

Starting from 2006, the EU RoHS Directive banned electrical and electronic equipment from entering the European market if it contained toxic levels of lead, cadmium, mercury, hexavalent chromium, polybrominated biphenyl (PBB) and polybrominated diphenyl ether (PBDE) flame retardants. This is applicable to lighting products including LED lamps and therefore it can be considered that LED lamps currently available on the market are not toxic. Nevertheless, as detected by [32] in the LEDs analysed in their study, the combined weight of all metals was approximately a third of the total LED weight; the remaining weight was from the plastic housing. Potential implications may arise from the plastic housing. Tests carried out at ALAB in Berlin [34] investigated the Volatile Organic Compounds (VOCs) emissions from various compact fluorescent lamps, and the results showed that all the lamps analysed released VOCs, including some that are considered carcinogenic, such as phenol. However, the investigation was carried out in a test chamber with a confined volume of 22.5 litres, which is relatively much smaller in relation to lamp size compared with a real room setting; therefore the VOC concentrations from such lamps in real room conditions would be negligible. Further independent studies have not been identified to back up the results of these tests, and no other information could be found as to VOC emissions from operating compact fluorescent lamps or LEDs. Therefore additional research is required to investigate potential health hazards caused by VOC emissions, particularly from LEDs incorporating plastic compounds.

Most gas discharge lamps contain mercury, which is needed for optimal energy efficiency and life cycle performance. European legislation limits the mercury content of discharge lamps, and recommendations exist on how to reduce exposure to mercury from broken lamps. However, LED lamps do not contain mercury and this makes them safer in case of accidental lamp breakage compared to discharge lamps, and compliant with European legislation.

\section{Skin exposure to LED lighting}

Radiation can cause skin damage. Exposure to radiation can also exacerbate existing skin conditions such as lupus [35]. Burns are caused by exposure to very high levels of visible or infrared radiation. The other types of skin damage (erythema, elastosis and cancers) are caused by exposure to UV radiation. Indoors, UV levels are much lower. The exposure to UV drops with the square of the distance from the lamp, so conventional ceilingmounted lamps are unlikely to cause problems. Whilst fluorescent tubes and CFLs can emit some UV radiation, LEDs emit little or no UV and would not cause this type of skin damage.

\section{Electric fields generated by LED lighting}

Lamps and control gear include electric and/or electronic components through which electric currents flow so that light output is initiated and maintained. These electric currents generate magnetic and electric fields of low frequency ( $50 \mathrm{~Hz}$ and harmonics thereof, eg $100 \mathrm{~Hz}, 150 \mathrm{~Hz}$ ) and high frequency (30 to $60 \mathrm{kHz}$ ) depending on the type of lamp and control gear [36]. Above certain intensities, the magnetic and electric fields may induce electrical currents in the human body that can stimulate the nerves and muscles at low frequency, or even cause tissue heating at high frequency [36, 37].

Improvements in lighting technology have enabled a reduction in electromagnetic fields. For LED lighting, several studies [38, 39] have shown that the intensities of magnetic fields generated by LED lamps were significantly below the limits recommended by the International Commission for Nonionizing Radiation Protection (ICNIRP). Therefore LED lighting does not appear to generate electric or magnetic fields that can damage human health. 


\section{Conclusion}

LEDs are directional by nature and hence can be very bright and glaring light sources. Very bright LEDs may cause retinal damage if viewed directly. Night-time exposure to bright LEDs rich in blue light can also alter the body clock and lead to various health problems. LEDs can exhibit flicker, which can cause headaches, eyestrain or epileptic seizures in some people. As European legislation bans electrical and electronic equipment that contains toxic levels of chemicals, LEDs currently available on the market should not contain chemicals above human toxicity levels. However, further research is needed to investigate potential health hazards caused by VOC emissions from LEDs that incorporate plastic compounds. On the other hand, LEDs also have benefits, as they do not contain mercury, emit little or no UV and do not generate electric or magnetic fields that can damage human health.

In order to minimise health risks and maximise health benefits of LED lighting, a number of measures should need to be considered by lighting designers and building owners and occupiers. Glare should be controlled using suitable shielding of high luminance parts of the lighting system or using luminaires that generate glare rating indices within standard recommendations. Bright light with a high blue content, such as that emitted by high power cold white LEDs, should be prevented from shining directly in occupants' eyes. LEDs should have suitable drivers and compatible lighting controls should be used in order to avoid flicker, and dimming should be considered to allow lower light levels in the evening. At the end of life, LEDs should be disposed of correctly by following local lamp recycling instructions.

\section{References}

[1] SLL. The SLL Lighting Handbook. London: Society of Light and Lighting; 2009.

[2] Ticleanu C et al. Lighting and health. Bracknell: IHS BRE Press; 2015.

[3] Stone PT. A model for the explanation of discomfort and pain in the eye caused by light. Lighting Research and Technology. 2009; 41:109-121.

[4] HSE. Lighting at work. HSG 38. Sudbury: HSE Books; 1997.

[5] SLL. The SLL Code for Lighting. London: Society of Light and Lighting; 2012.

[6] Werth L et al. Psychologische Befunde zu Licht und seiner Wirkung auf den Menschen - ein Überblick. Bauphysik. 2013; 35(3):193-204.

[7] Howarth PA et al. Discomfort from glare: The role of pupillary hippus. Lighting Research and Technology. 1993; 25(1):37-42.

[8] Cree. Eye safety with LED components. Cree; 2014. Available at: www.cree.com/xlamp_app_ notes/led_eye_safety

[9] Chamorro E et al. Effects of light-emitting diode radiations on human retinal pigment epithelial cells in vitro. Photochemistry and Photobiology. 2013; 89:468-473.

[10]Wilkins AJ et al. LED lighting flicker and potential health concerns: IEEE standard PAR1789 update. Atlanta: IEEE ECCE; 2010. p. 171-178.

[11]Bullough JD et al. Effects of flicker characteristics from solid-state lighting on detection, acceptability and comfort. Lighting Research and Technology. 2011; 43(3): 337-348.

[12] Wilkins AJ. Light right for sight - health and efficiency in lighting practice. Journal of the Illuminating Engineering Society. 1994; 23(2):138-156.

[13] IEEE. A review of the literature on light flicker: Ergonomics, biological attributes, potential health effects, and methods in which some LED lighting may introduce flicker. IEEE Standards Association; 2010. Available at: http://grouper.ieee.org/groups/1789/FlickerTR1_2_26_10.pdf

[14] Veitch JA and McColl SL. Full spectrum fluorescent lighting effects on people: A critical review. Ottawa: Institute for Research in Construction; 1993.

[15]DOE. Flicker: Solid-state lighting technology fact sheet. US Department of Energy, Building Technologies Office; 2013. Available at http://apps1.eere.energy.gov/buildings/publications/pdfs/ssl/flicker_fact-sheet.pdf

[16] IET. Code of practice for the application of LED lighting systems. London: Institution of Engineering and Technology; 2014.

[17] Kantermann T. Circadian biology: Sleep-styles shaped by light-styles. Current Biology. 2013; 23(16):R689R690.

[18] Gooley JJ et al. Exposure to room light before bedtime suppresses melatonin onset and shortens melatonin duration in humans. Journal of Clinical Endocrinology and Metabolism. 2011; 96(3):E463-E472. 
[19] Wood B et al. Light level and duration of exposure determine the impact of self-luminous tablets on melatonin suppression. Applied Ergonomics. 2013; 44:237-240.

[20] Figueiro MG et al. The impact of watching television on evening melatonin levels. Journal of the Society for Information Display. 2013; 21(10):417-421.

[21] Rea M et al. The potential of outdoor lighting for stimulating the human circadian system. Muldersdrift: EE Publishers; 2014. Available at: www.ee.co.za/article/potential-outdoor-lighting-stimulating-humancircadian-system.html

[22]DOE. Light at night: The latest science. Whitepaper. US Department of Energy, Solid-State Lighting Program; 2010. Available at http://apps1.eere.energy.gov/buildings/publications/pdfs/ssl/ssl_white paper_nov2010.pdf

[23] Cajochen C et al. High sensitivity of human melatonin, alertness, thermoregulation, and heart rate to short wavelength light. Journal of Clinical Endocrinology \& Metabolism. 2005; 90(3):1311-1316.

[24] West KE et al. Blue light from light-emitting diodes elicits a dose-dependent suppression of melatonin in humans. Journal of Applied Physiology. 2011; 110(3):619-626.

[25] Czeisler CA. Perspective: Casting light on sleep deficiency. Nature. 2013; 497:S13.

[26] Arendt J. Biological rhythms during residence in polar regions. Chronobiology International. 2012; 29(4):379-394.

[27] Glickman G et al. Light therapy for Seasonal Affective Disorder with Blue Narrow-Band Light-Emitting Diodes (LEDs). Biological Psychiatry. 2006; 59:502-507.

[28] Strong RE et al. Narrow-band blue-light treatment of seasonal affective disorder in adults and the influence of additional nonseasonal symptoms. Depression and Anxiety. 2009; 26:273-278.

[29] Osram. Life cycle analysis, 2014. Available at www.osram.com/osram_com/sustainability/sustainableproducts/life-cycle-analysis/index.jsp

[30] Osram. Life cycle assessment of illuminants: A comparison of light bulbs, compact fluorescent lamps and LED lamps. Regensburg: Osram Opto Semiconductors GmbH; 2009. Available at: www.energetica.eu/mediapool/99/993141/data/OSRAM_LED_LCA_Summary_November_2009.pdf

[31]DEFRA. Life cycle assessment of ultra-efficient lamps. London: Department for Environment, Food and Rural Affairs; 2009.

[32]Lim SR et al. Potential environmental impacts of Light-Emitting Diodes (LEDs): Metallic resources, toxicity, and hazardous waste classification. Environmental Science and Technology. 2011; 45(1):320-327.

[33]EC. Are waste LEDs hazardous? Science for Environment Policy, European Commission DG Environment News Alert Service, 229. Brussels: European Commission, February 2011.

[34]Büchel J. Bei sechs Produkten ging bald einmal das Licht aus. K-Tipp, 14, 7 September 2011.

[35] Moseley $\mathrm{H}$ and Ferguson. The risk to normal and photosensitive individuals from exposure to light from compact fluorescent lamps. Photodermatology, Photoimmunology \& Photomedicine. 2011; 27(3):131-137.

[36]EU. Light sensitivity. Scientific Committee on Emerging and Newly Identified Health Risks. Brussels: European Union; 2008.

[37]BAG. EMF Faktenblätter: Energiesparlampen. Bern: Bundesamt für Gesundheit 2014. Available at www.bag.admin.ch/themen/strahlung/00053/00673/02326/index.html?lang=de

[38] Nadakuduti J et al. Assessment of EM exposure of energy-saving bulbs \& possible mitigation strategies. Zürich, IT IS Foundation, 2010. Available at www.news.admin.ch/NSBSubscriber/message/ attachments/18707.pdf

[39]BSI. Assessment of lighting equipment related to human exposure to electromagnetic fields. PD IEC/TR 62493-1:2013. London: BSI, 2013. 